\title{
Fluctuation Electron Microscopy and Computational Structure Refinement for the Structure of Amorphous Materials
}

\author{
Jason Maldonis ${ }^{1}$, Pei Zhang ${ }^{1}$, Li He ${ }^{1}$, Ankit Gujral ${ }^{2}$, Mark D. Ediger ${ }^{2}$, Paul M. Voyles ${ }^{1}$ \\ 1. Department of Materials Science and Engineering, University of Wisconsin-Madison, Madison, WI \\ 53706, USA \\ 2. Department of Chemistry, University of Wisconsin-Madison, Madison, WI 53706, USA
}

Fluctuation electron microscopy (FEM) uses systematic, scanning coherent electron nanodiffraction to measure the medium-range order (MRO) structure in amorphous materials [1]. MRO covers the third to fifth coordination shell, which is $\sim 1$ nanometer for inorganic materials like metallic glasses and $\sim 5 \mathrm{~nm}$ for organic materials such as a small molecule glasses. Figure 1(a) shows a typical speckle pattern from coherent nanodiffraction. Each speckle arises from a collection of atoms in the sample with sufficient structural order to diffract in a preferred direction. FEM measures spatial fluctuations in the intensity of these speckles from place to place using the normalized variance, $V(k)=\left\langle I^{2}(k)\right\rangle /\langle I(k)\rangle^{2}-1$.

The $k$ position of peaks in $V$ is related to preferred interatomic distances within the ordered regions, and the magnitude of the peaks is controlled by their size, density, and degree of internal structural order. In some cases, this is sufficient to connect FEM data to structural models, but for more complex systems, we have developed a computational structure refinement scheme based on hybrid reverse Monte Carlo (HRMC) modeling [2]. HRMC creates a computer model that minimizes the sum of the system energy and the $\chi^{2}$ deviation between experiments and simulations of FEM data. The energy constrains the chemical- and short-range order, and the FEM constrains the MRO.

We have applied FEM to study the structure of bulk metallic glasses and molecular glass thin films. Figure 1(b) shows a typical FEM measurement from a $\mathrm{Zr}_{50} \mathrm{Cu}_{35} \mathrm{Al}_{15}$ bulk metallic glass and the corresponding simulation from the HRMC model refined against that data. In two different prototypical metallic glass alloys systems, we have discovered a mix of two structure types at medium range. One structure has approximate crystalline rotational symmetry and the other does not. As shown in Figure 2, in Zr-Cu-Al, a prototype all-metal glass, the crystal-like structure has 6-fold rotational symmetry. The non-crystal-like structure has icosahedral short-range order. Pd-Si, a prototype metal-metalloid glass, has different local structures but the same competition between two types of MRO. In Zr-Cu-Al, the stability of the crystallike structure is correlated with reduced glass-forming ability [3].

Figure 3 shows FEM data from glassy thin films of the small molecule indomethacin (IMC). Because the relevant MRO length scale is intermolecular instead of interatomic, these data were collected with a larger, less convergent electron probe $(13 \mathrm{~nm}$, vs $2 \mathrm{~nm}$ for the metallic glass data) and over much smaller scattering angles. IMC thin films deposited on a substrate held at $85 \%$ of the glass transition temperature have much higher thermodynamic and kinetic stability than IMC thin films rendered glassy by quenching from the liquid [4]. The FEM $V(k)$ from the quenched glass IMC primarily shows peaks at $k$ positions near features in the structure factor for the $\alpha$-IMC crystal structure, indicating similar intermolecular packing in the glass and the crystal. Ultrastable IMC has a lower- $k$ peak, indicating intermolecular ordering at longer length scales not seen in any reported IMC crystal structure [5]. 
References:

[1] M. M. J. Treacy et al., Rep. Prog. Phys. 68, 2899 (2005)

[2] J. Hwang, et al., Phys. Rev. Lett. 108, 195505 (2012)

[3] P. Zhang et al. Acta Mat., to appear (2016)

[4] S. F. Swallen et al. Science 315, 353 (2007)

[5] Research on metallic glasses was supported by NSF DMR-1506564. Research on molecular glasses was supported by the NSF through the University of Wisconsin MRSEC (DMR-1121288).
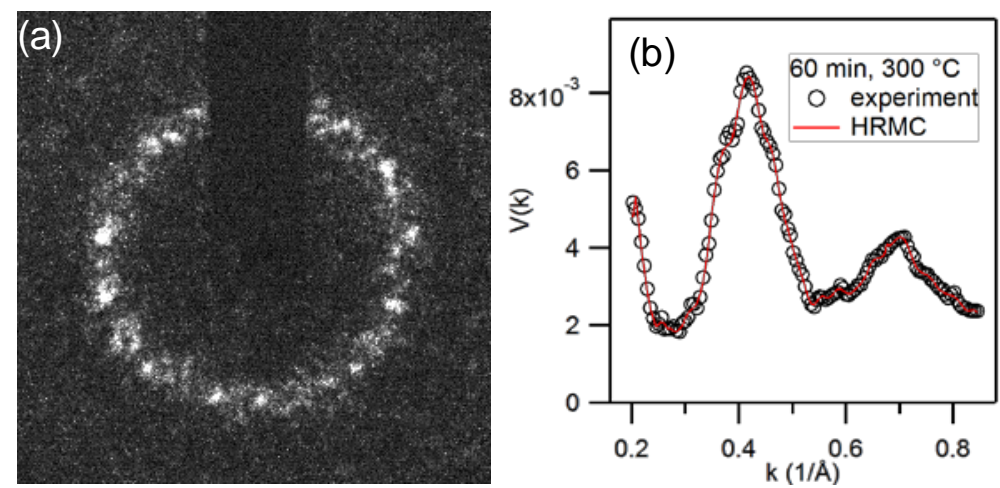

Figure 1: (a) typical nanodiffraction pattern from a metallic glass. (b) FEM $V(k)$ data from $\mathrm{Zr}_{50} \mathrm{Cu}_{35} \mathrm{Al}_{15}$ metallic glass after annealing, with the simulated $V(k)$ from the refined HRMC model.

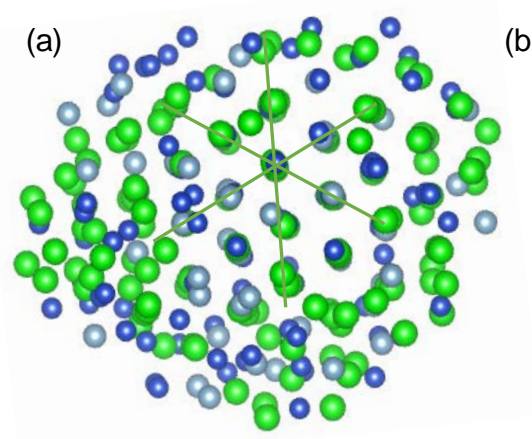

(b)

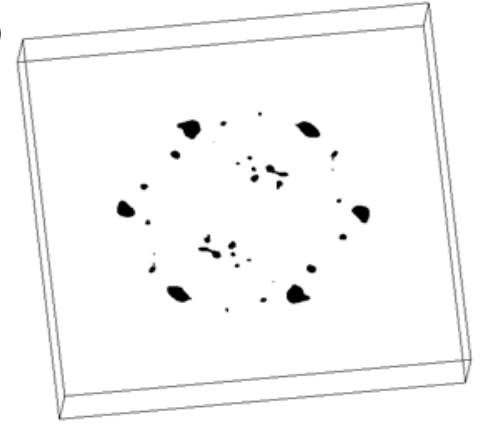

Figure 2: (a) central region of the HRMC model, emphasizing the crystal-like region with 6-fold rotation symmetry. (b) An isosurface plot of the calculated 3D reciprocal space of the same model showing the corresponding six-fold ring of spots.
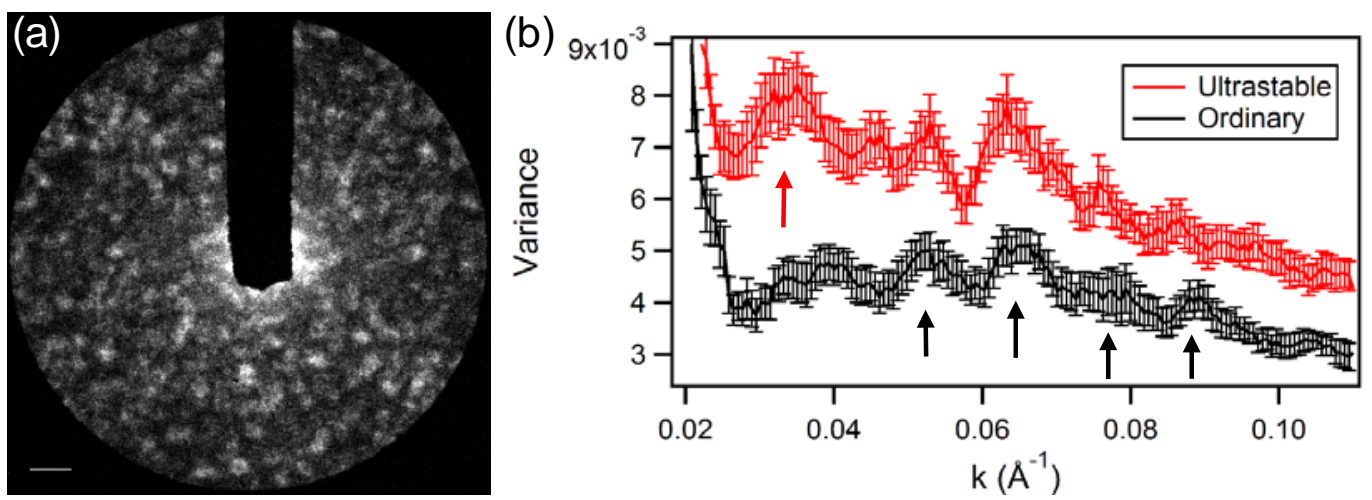

Figure 3: (a) typical speckle nanodiffraction pattern from an ultrastable IMC thin film (b) FEM $V(k)$ data comparing the ultrastable glass and normal glass state of IMC. 